\title{
Lymphoma presenting as mononeuritis multiplex
}

\author{
Alison C. ReID \\ M.B. B.S., F.R.A.C.P., M.R.C.P. \\ I. BONE \\ M.B., Ch.B., M.R.C.P. \\ University Department of Neurology, Institute of Neurological Sciences,
Southern General Hospital, Glasgow G51 4TF
}

\begin{abstract}
Summary
A case is reported of mononeuritis multiplex, the peripheral nerve lesions being caused by metastatic deposits of lymphoma in muscle. The patient also had a past history of a facial nerve palsy and the GuillainBarré syndrome. This case portrays an interesting spectrum of unusual manifestations of neoplastic disease - both with regard to metastatic spread and peripheral neurological involvement.

\section{Introduction}

Despite the rich blood supply, clinically detectable metastases to muscle are very uncommon and the reason for this is poorly understood. Direct metastatic spread of malignant cells frequently takes place to the brain, spinal cord and nerve roots, where compression and infiltration of nervous tissue may occur. Involvement of peripheral nerves usually occurs from an indirect neoplastic effect which takes the form of a symmetrical peripheral neuropathy (Denny-Brown, 1948). A most unusual case is described of mononeuritis multiplex due to metastatic spread of non-Hodgkin's lymphoma to muscle, with direct involvement of peripheral nerves.
\end{abstract}

\section{Case report}

In May 1979 a 63-year-old woman suddenly developed painless right wrist drop and 4 weeks later weakness of the left leg with foot drop. There was no history of trauma and she had no paraesthesia or numbness. Her general health was apparently excellent apart from long-standing asthma and hypertension.

She had developed a left facial nerve palsy 2.5 years previously, which had been sudden in onset and which had cleared spontaneously. Two years before, she had been admitted to hospital with an acute onset of a sensori-motor peripheral neuropathy. Diagnosis of a Guillain-Barré syndrome had been made and this had resolved completely over the next 4 weeks.

She was obese with a mild residual left lower motor neuron facial weakness. There was reduced power in the right arm in a radial nerve distribution and weakness of the left foot and sensory disturbance in the distribution of the deep peroneal nerve. There was a large mass in the right deltoid and another hard mass in the lateral head of the left gastrocnemius. A firm left supraclavicular lymph node was palpable but there was no generalized lymphadenopathy.

Haemoglobin was $13.6 \mathrm{~g} / \mathrm{dl}$ and the WBC $3.7 \times$ $10^{9} / 1$ with a mild neutropenia and moderate lymphopenia. ESR was $30 \mathrm{~mm}$ in the first hour (Westergren). Serum urea, electrolytes and liver function tests were normal. Serum LDH was grossly elevated at 1560 i.u./l and electrophoresis of iso-enzymes indicated this was of muscle origin. Serum immunoelectrophoresis showed normal IgG but low levels of IgA and IgM. Chest radiograph was normal. Nerve conduction studies produced no elicitable response from the right radial and left deep peroneal nerves. Biopsy and histology of the deltoid mass revealed a cellular malignant tumour with numerous mitotic figures. Bone marrow aspiration confirmed the presence of poorly differentiated non-Hodgkin's lymphoma.

\section{Discussion}

Metastases of malignant neoplasms to skeletal muscle are infrequent. In a survey of 500 postmortems of patients who had died of cancer, Willis (1948) observed metastases to skeletal muscle in only 4 cases $(0.8 \%) ; 2$ of epidermoid carcinoma of the head and neck and 2 of thyroid carcinoma. Pearson (1959) detected metastatic tumour at post-mortem in 6 cases from a total of 38 patients with malignancy at the time of death. There were 3 lymphomas, and carcinomas of the ampulla of Vater, stomach and male breast. The higher incidence of $16 \%$ skeletal muscle metastases in this series was attributed to the fact that a larger number of muscles were sampled in each patient. Buerger and Monteleone (1966) in a post-mortem series of patients with leukaemia and 
lymphoma found skeletal muscle involvement in 43 of 82 cases $(52 \%)$ indicating that tumours of lymphoid tissue have a greater tendency to involve muscle.

Tumour cells are usually seen as perimysial growths adjacent to a blood vessel. The metastatic growth often destroys adjacent muscle fibres by direct invasion. Tumour cells have also been found in skeletal muscle syncitia suggesting an intracellular route for the spread of malignant tumour within muscle (Slatkin and Pearson, 1976). Ultrastructural studies (Carr, McGinty and Norris, 1975) have actually demonstrated neoplastic cells penetrating the sarcolemma and invading skeletal muscle syncitia accompanied by polymorphs and macrophages. The syncitia disintegrate and the myofibrillar debris is phagocytosed by macrophages.

It is important to note that in the above 3 postmortems, neoplastic involvement in muscle was only recognized microscopically and not macroscopically. The number of reports of discrete metastatic masses detected in muscle are surprisingly few. Single cases of squamous cell carcinoma of the lung (Pellegrini, 1979), carcinoma of the colon (Laurence and Murray, 1970) and carcinoma of the pancreas (Mulsow, 1943) presenting as discrete muscle masses have been reported. The factors responsible for this low frequency of skeletal muscle metastatic deposits are not understood. The present case was remarkable in that there were not only 2 large metastatic deposits, but at sites resulting in nerve compression and infiltration. The mass in the lateral head of the left gastrocnemius was closely situated to the deep peroneal nerve, whilst the mass in the right deltoid was some distance from the radial groove. It must therefore be surmised that neoplastic cells from the latter mass were extensively infiltrating into nearby muscles. One might even speculate that perhaps the nerves were primarily involved with secondary spread to muscle. Infiltration of nerves has been reported in Hodgkin's and non-Hodgkin's lymphoma; longitudinal invasion of the perineurium and endoneurium causing axonal degeneration and segmental demyelination (Henson and Urich, 1970).

It is of interest to note that mononeuritis multiplex has recently been described from 3 patients who only subsequently were found to have oat cell carcinoma, lymphoma and liposarcoma (Johnson et al., 1979). This was thought to be a new kind of remote effect of cancer on the peripheral nervous system, as nerve biopsy showed active wallerian degeneration with vasculitis, but no invasion by malignant cells.

This patient also had a history of the GuillainBarré syndrome which is known occasionally to occur in association with lymphomas (Lisak et al., 1977; Currie et al., 1970). Acute idiopathic polyneuritis is widely believed to be an immunologically mediated disease caused by delayed hypersensitivity to peripheral nervous system myelin antigens (Asbury, Arnason and Adams, 1969). The association with lymphomas, however, in which there is commonly a degree of immunosuppression, suggests that partial immune deficiency could serve as a triggering factor in autoimmune neuropathy (Lisak et al., 1977).

The aetiology of the transient facial nerve palsy in the present case is not clear. Since almost complete resolution of the weakness occurred, direct neoplastic invasion of the nerve or a non-metastatic effect was unlikely. The history suggests that it was most probably a fortuitous Bell's palsy, unrelated to the latent lymphoma.

\section{References}

Asbury, A.K., Arnason, B.G.W. \& Adams, R.D. (1969) The inflammatory lesion in idiopathic polyneuritis: its role in pathogenesis. Medicine, 48, 173.

Buerger, L.G. \& Monteleone, P.N. (1966) Leukemiclymphomatous infiltration of skeletal muscle. Cancer, 19, 1416.

Carr, I., McGinty, F. \& Norris, P. (1975) The fine structure of neoplastic invasion: invasion of liver, skeletal muscle and lymphatic vessels by the $\mathrm{Rd} / 3$ tumour. Journal of Pathology, 118, 91.

Currie, S., Henson, R.A., Morgan, H.G. \& Poole, A.J. (1970) The incidence of the non-metastatic neurological syndromes of obscure origin in the reticuloses. Brain, 93, 629.

DenNy-Brown, D. (1948) Primary sensory neuropathy with muscular changes associated with carcinoma. Journal of Neurology, Neurosurgery and Psychiatry, 11, 73.

Henson, R.A. \& URICH, H. (1970) Peripheral neuropathy associated with malignant disease. In: Handbook of Clinical Neurology, Vol. 8, Ch. 9 (Ed by Vinken, P.J. \& Bruyn, C.W.), p. 131. North-Holland, Amsterdam.

Johnson, P.C., Rolak, L.A., Hamilton, R.H. \& Laguna, J.F. (1979) Paraneoplastic vasculitis of nerve: A remote effect of cancer. Annals of Neurology, 5, 437.

Laurence, A.E. \& Murray, A.J. (1970) Metastasis in skeletal muscle secondary to carcinoma of the colon. British Journal of Surgery, 57, 529.

LisaK, R.P., Mitchell, M., Zweiman, B., OrRechio, E. \& AsBury, A.K. (1977) Guillain-Barré syndrome and Hodgkin's disease: three cases with immunological studies. Annals of Neurology, 1, 72.

Mulsow, F.W. (1943) Metastatic carcinoma of skeletal muscles. Archives of Pathology, 35, 112.

Pearson, C.M. (1959) Incidence and type of pathologic alterations observed in muscle in a routine autopsy survey. Neurology, 9, 757.

Pellegrini, A.E. (1979) Carcinoma of the lung occurring as a skeletal muscle mass (letter). Archives of Surgery, 114, 550.

Slatkin, D.N. \& Pearson, J. (1976) Intramyofiber metastases in skeletal muscle. Human Pathology, 7, 347.

Willis, R.A. (1948) Pathology of Tumors. C. V. Mosby, St Louis. 